\title{
NIVELES DE HEMOGLOBINA EN GESTANTES ATENDIDAS EN ESTABLECIMIENTOS DEL MINISTERIO DE SALUD DEL PERÚ, 2011
}

\author{
Oscar Munares-García1,2,a, Guillermo Gómez-Guizado1,b, Juan Barboza-Del Carpio,c, \\ José Sánchez-Abanto1,d
}

\section{RESUMEN}

Objetivos. Determinar los niveles de hemoglobina y la prevalencia de anemia en gestantes atendidas en los establecimientos del Ministerio de Salud a nivel nacional. Materiales y métodos. Estudio transversal donde se analizó la base de datos del Sistema de Información del Estado Nutricional del Niño menor de 5 años y de la Gestante (SIEN). Se incluyó 287691 registros de gestantes evaluadas en establecimientos del Ministerio de Salud del Perú en 2011, se analizaron los niveles de hemoglobina corregida a la altura, edad, edad gestacional, altitud a nivel del mar y prevalencia de anemia (leve, moderada y grave). Se aplicaron estadísticas descriptivas y chi cuadrado. Resultados. La prevalencia a nivel nacional de anemia en la gestante fue de $28,0 \%$ siendo anemia leve de $25,1 \%$, moderada de $2,6 \%$ y grave de $0,2 \%$. Los niveles de hemoglobina son mayores en mujeres con mayor edad y menores durante los primeros meses de gestación, la frecuencia de anemia decrece con la altitud. Asimismo, la prevalencia es mayor en departamentos de la sierra. Huancavelica fue el departamento con mayor prevalencia de anemia (53,6\%), seguido de Puno con 51,0\%. Conclusiones. Los niveles de hemoglobina son mayores conforme la edad materna es mayor, y menores conforme el trimestre de gestación y altitud. Huancavelica tiene la mayor prevalencia de anemia en gestantes.

Palabras clave: Anemia; Embarazo; Hemoglobinas; Estado nutricional; Establecimientos de salud (fuente: DeCS BIREME).

\section{HEMOGLOBIN LEVELS IN PREGNANT WOMEN SEEN IN HEALTH CARE CENTERS OF THE PERUVIAN MINISTRY OF HEALTH, 2011}

\begin{abstract}
Objectives. Determine hemoglobin levels and prevalence of anemia in pregnant women seen in health care centers of the Ministry of Health at national level. Materials and methods. Cross-cut study where the database of the Information System on the Nutritional Health of Children under 5 and Pregnant Women (SIEN) were analyzed. 287691 records of pregnant women examined at the health care centers of the Peruvian Ministry of Health in 2011 were included, hemoglobin levels corrected by height, age, gestational age, altitude and prevalence of anemia (light, moderate and serious) were analyzed. Descriptive statistics and the chi-square method were used. Results. Nationwide prevalence of anemia in pregnant women was $28.0 \%$, with mild anemia being at $25.1 \%$, moderate anemia at $2.6 \%$ and severe anemia at $0.2 \%$. Hemoglobin levels are higher in older and younger women during the first months of pregnancy, prevalence of anemia decreases with altitude. Furthermore, prevalence is higher in the Highland regions. Huancavelica was the region with higher prevalence of anemia (53.6\%), followed by Puno with 51.0\%. Conclusions. Hemoglobin levels get higher as the mother gets older, and they go down in keeping with the gestation trimester and altitude. Huancavelica has the highest prevalence of anemia in pregnant women.
\end{abstract}

Key words: Anemia; Pregnancy; Hemoglobins; Nutritional status; Health facilities (source: MeSH NLM).

Centro Nacional de Alimentación y Nutrición, Instituto Nacional de Salud. Lima, Perú.

Departamento Académico de Obstetricia, Facultad de Medicina Humana, Universidad Nacional Mayor de San Marcos. Lima, Perú.

Licenciado en Obstetricia magister en Salud Pública; ${ }^{\mathrm{b}}$ médico cirujano especialista en Epidemiologia de Campo; ${ }^{\mathrm{c}}$ nutricionista; ${ }^{\mathrm{d}}$ nutricionista magister en Nutrición Recibido: 20-06-12 Aprobado: 29-08-12

* Parte de este artículo se basa en el informe técnico "Anemia en gestantes del Perú y provincias con comunidades nativas 2011" disponible en la página web del Centro Nacional de Alimentación y Nutrición del Instituto Nacional de Salud de Perú.

Citar como: Munares-García O, Gómez-Guizado G, Barboza-Del Carpio J, Sánchez-Abanto J. Niveles de hemoglobina en gestantes atendidas en establecimientos del Ministerio de Salud del Perú, 2011. Rev Peru Med Exp Salud Publica. 2012;29(3):329-36. 


\section{INTRODUCCIÓN}

En el Perú, el $19 \%$ de las mujeres entre 12 y 49 años padece de anemia, siendo mayor en el área rural (22\%) y en zonas con pobreza extrema $(26 \%){ }^{(1)}$. Durante el embarazo, las necesidades de hierro se incrementan hasta tres veces a consecuencia de las pérdidas basales, del aumento de masa de glóbulos rojos y del crecimiento del feto, la placenta y tejidos maternos asociados ${ }^{(2,3)}$; la concentración de hemoglobina $(\mathrm{Hb})$ varía con la altitud, por ello, la Organización Mundial de la Salud (OMS) recomienda establecer como anemia durante la gestación valores menores a $11 \mathrm{~g} / \mathrm{dL}$. Debido a este incremento de necesidades de hierro, la anemia ferropénica es la enfermedad hematológica de mayor prevalencia en la embarazada (4) y está presente en el 30 al $70 \%{ }^{(5)}$ de las gestantes; las cuales presentan variaciones que disminuyen su concentración promedio ${ }^{(6)}$. Según la Encuesta Demográfica y de Salud Familiar (ENDES) en su versión 2011, la prevalencia nacional de anemia en gestantes fue $27,8 \%$ ( 7 ; ; otro estudio realizado en establecimientos de salud del Perú determinó una menor proporción de anemia en gestantes de la costa $(25,8 \%)$ y selva baja $(26,2 \%)^{(8)}$.

Según el censo de 2007, el $42,2 \%$ de la población en el Perú tiene afiliación a algún tipo de seguro de salud: $18,4 \%$ al sistema de Seguro Integral de Salud del Ministerio de Salud (SIS); $17,9 \%$ en EsSalud y $5,9 \%$ a otros seguros públicos o privados, quedando un restante $57,8 \%$ sin afiliación ${ }^{(9)}$. Estas gestantes son atendidas principalmente en establecimientos del Ministerio de Salud, cuya cobertura para el año 2005 fue de $62,7 \%{ }^{(10)}$. Durante la gestación la estrategia de vigilancia se realiza en el control prenatal, que conjunto con el parto institucional son las principales estrategias para reducir la morbimortalidad materna y neonatal. Durante el control prenatal se realiza la detección y manejo de la anemia durante el embarazo, determinando los niveles de hemoglobina en la primera consulta, de preferencia en el primer trimestre. Según ENDES el $74,9 \%{ }^{(7)}$ de las gestantes tuvo su control prenatal en los primeros cuatro meses de embarazo; durante las consultas se ofrece tratamiento con sulfato ferroso para volver a determinar el nivel de hemoglobina en la sexta consulta entre las 37 a 40 semanas ${ }^{(11)}$.

Al realizar la revisión de estudios con representatividad nacional o realizados en establecimientos de salud, no se encuentran datos actualizados de anemia en la gestación según grupos de edad, trimestre de gestación, altitud a nivel del mar, regiones de Perú, ni tampoco como referencia el último dosaje de hemoglobina realizada a la gestante. Por ello, el objetivo de este estudio fue determinar los niveles de hemoglobina y la prevalencia de anemia en gestantes atendidas en los establecimientos del Ministerio de Salud, según edad materna, trimestre de gestación, altitud a nivel del mar y por departamentos.

\section{MATERIAL Y MÉTODOS}

\section{DISEÑO Y POBLACIÓN DE ESTUDIO}

Estudio transversal donde se analizó la base de datos del Sistema de Información del Estado Nutricional del Niño (SIEN) del Centro Nacional de Alimentación y Nutrición (CENAN) del Instituto Nacional de Salud del Perú (INS). En esta base de datos se encuentran los registros de

Tabla 1. Características generales de las gestantes atendidas en establecimientos del Ministerio de Salud del Perú, 2011.

\begin{tabular}{|c|c|c|}
\hline Características & N. ${ }^{\circ}$ & $\%$ \\
\hline \multicolumn{3}{|l|}{ Edad (años) } \\
\hline $10-15$ & 6657 & 2,3 \\
\hline $16-35$ & 253555 & 88,1 \\
\hline $36-45$ & 27479 & 9,6 \\
\hline \multicolumn{3}{|l|}{ Trimestre } \\
\hline Primer & 26123 & 9,1 \\
\hline Segundo & 99737 & 34,7 \\
\hline Tercer & 161831 & 56,3 \\
\hline \multicolumn{3}{|l|}{ Altitud (m) } \\
\hline$<1000$ & 161660 & 55,6 \\
\hline $1001-1500$ & 35181 & 3,7 \\
\hline $1501-2500$ & 25876 & 8,9 \\
\hline $2501-3500$ & 59932 & 20,8 \\
\hline$>3500$ & 30918 & 10,7 \\
\hline \multicolumn{3}{|l|}{ Departamento } \\
\hline Amazonas & 4013 & 1,4 \\
\hline Ancash & 14064 & 4,9 \\
\hline Apurímac & 6445 & 2,2 \\
\hline Arequipa & 13501 & 4,7 \\
\hline Ayacucho & 12177 & 4,2 \\
\hline Cajamarca & 15896 & 5,5 \\
\hline Cusco & 19831 & 6,9 \\
\hline Huancavelica & 6275 & 2,2 \\
\hline Huánuco & 16384 & 5,7 \\
\hline Ica & 7700 & 2,7 \\
\hline Junín & 14419 & 5,0 \\
\hline La Libertad & 15205 & 5,3 \\
\hline Lambayeque & 8978 & 3,1 \\
\hline Lima & 67168 & 23,3 \\
\hline Loreto & 11565 & 4,0 \\
\hline Madre de Dios & 2054 & 0,7 \\
\hline Moquegua & 1030 & 0,4 \\
\hline Pasco & 4483 & 1,6 \\
\hline Piura & 11055 & 3,8 \\
\hline Puno & 13250 & 4,6 \\
\hline San Martín & 9475 & 3,3 \\
\hline Tacna & 2385 & 0,8 \\
\hline Tumbes & 2951 & 1,0 \\
\hline Ucayali & 7387 & 2,6 \\
\hline \multicolumn{3}{|l|}{ Establecimiento de salud } \\
\hline Centro de salud & 133521 & 46,4 \\
\hline Puesto de salud & 120200 & 41,8 \\
\hline Hospital & 33970 & 11,8 \\
\hline TOTAL & 287691 & 100,0 \\
\hline
\end{tabular}

Fuente: Sistema de Información del Estado Nutricional del Niño (SIEN); del Instituto Nacional de Salud. 


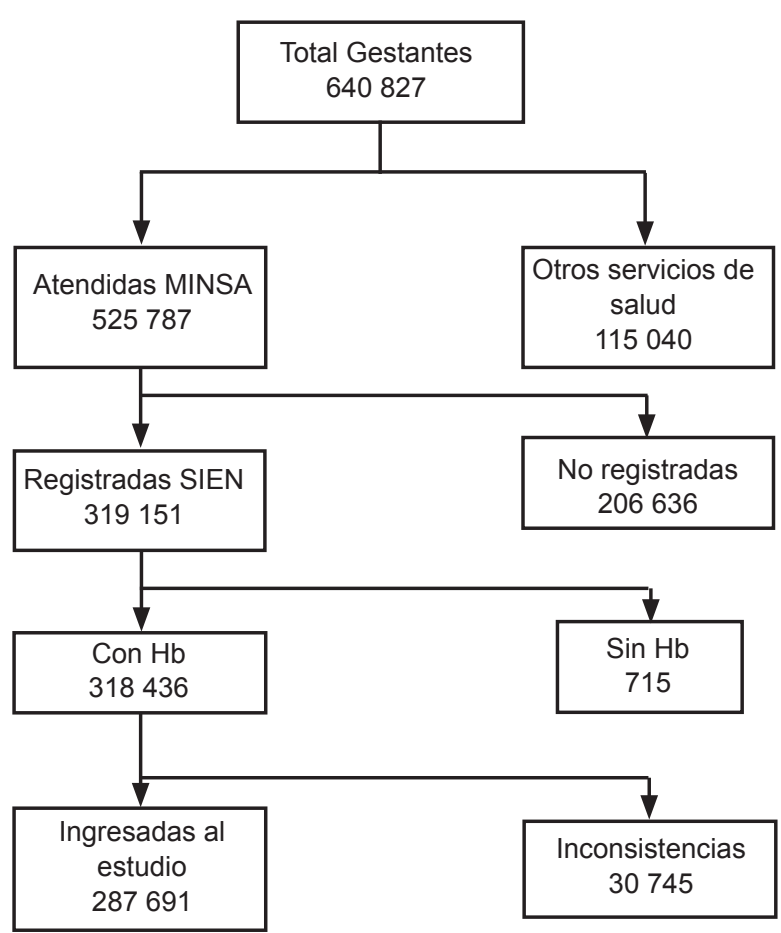

Figura 1. Estimación de la población de gestantes en Perú e incluidas en el estudio

* Estimado a partir del censo poblacional realizado el 2007 (INEI, Perú) MINSA: establecimientos del Ministerio de Salud.

SIEN: Sistema de Información del Estado Nutricional del Niño; del Instituto Nacional de Salud.

$\mathrm{Hb}$ : hemoglobina.

análisis de hemoglobina de gestantes que acudieron a su control prenatal en establecimientos de salud del Ministerio de Salud del Perú (5423 establecimientos divididos en 97 hospitales; 1113 centros de salud y 4213 puestos de salud, distribuidos de acuerdo a la Tabla 1; para el año 2011 fueron 318436 registros de gestantes, de ellas se incluyeron 287691 (90,3\%), excluyéndose registros inconsistentes y vacíos, de acuerdo al flujograma presentado en la Figura 1.

\section{PROCESAMIENTO DE LOS DATOS}

Durante el control prenatal, los profesionales solicitan a la gestante el dosaje de hemoglobina, una vez obtenido, se informa el resultado del laboratorio, tanto en la historia clínica y en el formato de registro SIEN por el personal que realiza la consulta prenatal; en este registro se incluye el lugar de procedencia (localidad) de la gestante y la altitud de dicha localidad (si no incluye el dato de localidad se aplica la altitud de la capital del distrito de procedencia y se ajusta si se encuentra a $1000 \mathrm{~m}$ de altitud o más) ${ }^{(12)}$. Luego los formatos pasan al punto de digitación, pudiendo ser el mismo establecimiento u otro ya convenido, este incorpora al sistema SIEN la información que luego es consolidada mensualmente en todos los puntos de digitación, enviada a la microrred de salud donde realizan la consolidación de la información de los establecimientos, luego se remite a la red, que lo envían a la dirección regional de salud y, finalmente, toda la información es enviada al CENAN. Cada punto de digitación realiza control de calidad sobre registros incompletos, datos inconsistentes o vacíos realizados por los puntos de digitación para reducir errores. Los informes de hemoglobina ajustada a la altura son realizados automáticamente por el sistema SIEN.

De los datos registrados, se analizó la edad (años); edad gestacional (por semanas y trimestres: primer 1-14 semanas, segundo 15-28 semanas y tercero 29-41 semanas 6 días); altitud a nivel del mar; departamentos del Perú (Amazonas, Ancash, Apurímac, Arequipa, Ayacucho, Cajamarca, Cusco, Huancavelica, Huánuco, Ica, Junín, La Libertad, Lambayeque, Lima, Loreto, Madre de Dios, Moquegua, Pasco, Piura, Puno, San Martín, Tacna, Tumbes, Ucayali) y valor de la hemoglobina ( $\mathrm{g} / \mathrm{dL})$.

La hemoglobina fue solicitada, al menos, en el primer control prenatal realizado a la gestante y durante el último trimestre de acuerdo con lo estipulado por el Ministerio de Salud ${ }^{(7)}$. Se determinó anemia si el resultado de hemoglobina fue $<11 \mathrm{~g} / \mathrm{dL}$. De acuerdo a la clasificación de la Organización Mundial de la Salud ${ }^{(9)}$ se clasificó en anemia leve ( $\mathrm{Hb}$ de 10-10,9 g/dL); moderada ( $\mathrm{Hb}$ de $7-9,9 \mathrm{~g} / \mathrm{dL})$ y grave $(\mathrm{Hb}<7 \mathrm{~g} / \mathrm{dL})$. Los valores de hemoglobina fueron ajustados a la altitud a nivel del mar.

\section{ANÁLISIS ESTADÍSTICO}

Se empleó el programa estadístico SPSS versión 17.0 para el análisis de los resultados de los niveles de hemoglobina y las prevalencias respectivas. En las variables cuantitativas como la hemoglobina se aplicó promedio, desviación estándar y error típico al $5 \%$. Para las variables cualitativas se aplicó la distribución de frecuencias y las proporciones como determinación de prevalencia. La edad se clasificó en tres rangos según riesgo para la gestación: 10-15 años; 16-35 años $y>36$ años, la edad gestacional fue dividida en primer trimestre (1-14 semanas), segundo (15-28 semanas) y tercero (29-41 semanas 6 días); la altitud en metros sobre el nivel del mar, se clasificó en $<1000$ metros de altitud; 1000-1500 metros de altitud; 1501-2500 metros de altitud; 2501-3500 metros de altitud, y $>3500$ metros de altitud. Para la determinación de la homogeneidad de las varianzas en la hemoglobina se aplicó chi cuadrado de Bartlett, en las variables cualitativas se aplicó el estadístico chi cuadrado de Pearson con un nivel de significación estadístico menor al 0,05. 
El estudio forma parte del Sistema de vigilancia del CENAN, y los datos provienen de actividades de rutina en la atención a las gestantes, las cuales obtuvieron los resultados de manos del profesional que las atendió $y$, cuando correspondía, recibieron tratamiento según protocolo. Este fue el motivo por el que no se solicitó la aprobación del comité de ética; no obstante, la base de datos no incluye nombres de participantes por lo que su identificación es solo a través del número de historia clínica y se mantuvo la confidencialidad de los datos.

\section{RESULTADOS}

La edad promedio de las gestantes estudiadas fue de $25,5 \pm 6,7$ años; la edad gestacional fue $28,1 \pm 8,5$ semanas; la talla fue de $1,51 \pm 0,55 \mathrm{~m}$, y el nivel de hemoglobina fue de $11,5 \pm 1,3 \mathrm{~g} / \mathrm{dL}$. El promedio de $\mathrm{Hb}$ entre los 10 a 15 años fue de $11,4 \mathrm{~g} / \mathrm{dL}$ y entre los 36 a 45 años fue de 11,6 $\mathrm{g} / \mathrm{dL}$. En el primer trimestre fue de $12,1 \mathrm{~g} / \mathrm{dL}$ y en el tercer trimestre fue de $11,5 \mathrm{~g} / \mathrm{dL}$. Conforme la altitud, el promedio a $<1000 \mathrm{~m}$ de altitud fue de $11,7 \mathrm{~g} / \mathrm{dL}$ y entre 3500 a $4000 \mathrm{~m}$ de altitud fue de $10,7 \mathrm{~g} / \mathrm{dL}$. El departamento de Lambayeque fue el de mayor promedio con $12,1 \mathrm{~g} / \mathrm{dL}$ y Cusco el de menor con 10,8 g/dL (Figura 2).

Se observa un leve incremento de los niveles promedio de hemoglobina conforme aumenta la edad materna; por otro lado, se observa un menor nivel del promedio de hemoglobina de acuerdo a los trimestres de gestación, y los niveles de hemoglobina son menores paulatinamente conforme el rango de altitud es mayor; por lo que se puede observar que en las gestantes que viven a menos de $1000 \mathrm{~m}$ de altitud el promedio es mayor

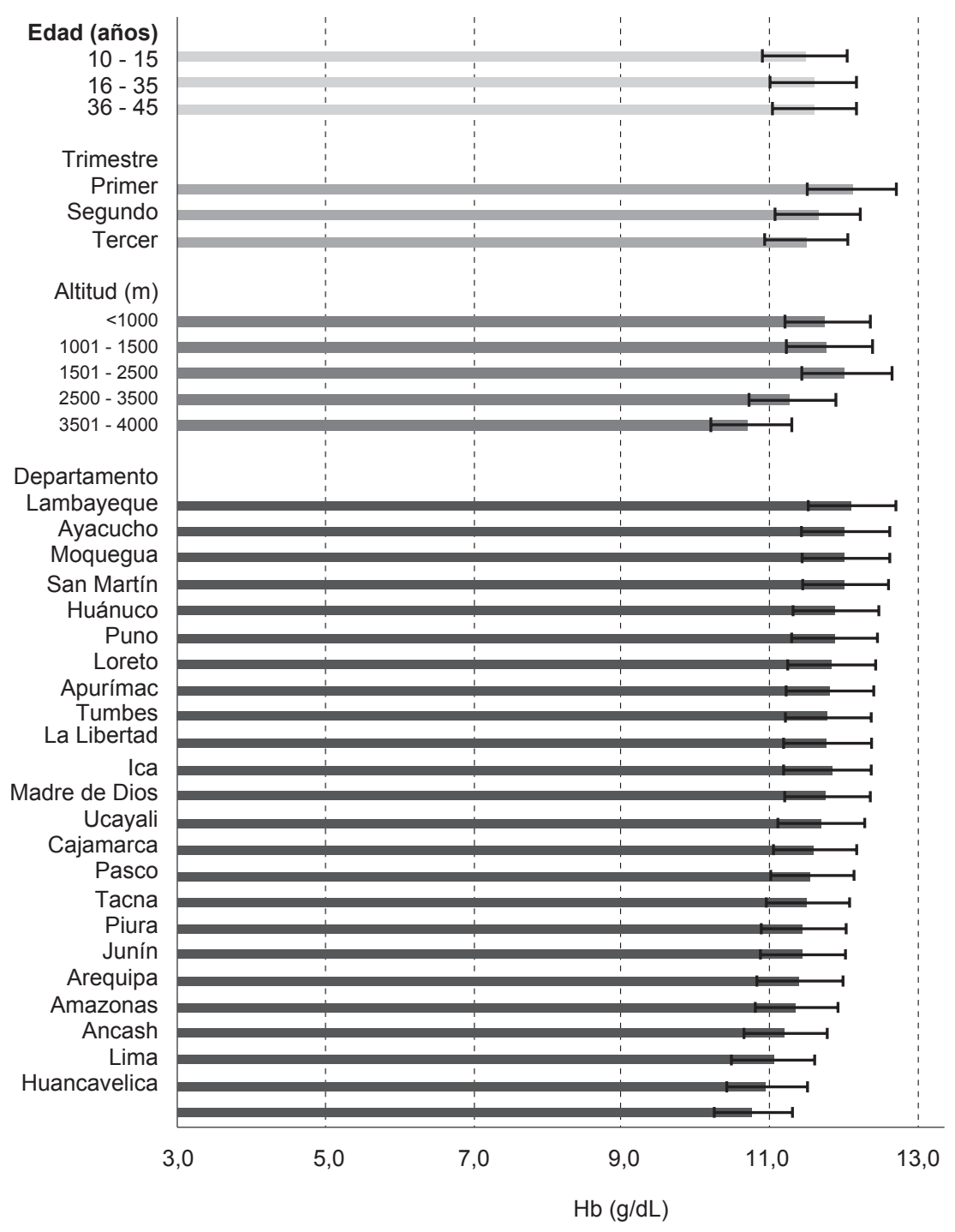

Figura 2. Valores de hemoglobina en gestantes atendidas en establecimientos del Ministerio de Salud del Perú, 2011. 
que aquellas que residen a más de $3500 \mathrm{~m}$ de altitud. Con respecto a los departamentos del Perú, se observa que en casi todos los promedios supera los $11 \mathrm{~g} / \mathrm{dL}$, exceptuando los departamentos de Lima, Huancavelica y Cusco que presentan cifras promedio menores a $11 \mathrm{~g} / \mathrm{dL}$. La prueba estadística confirmó que las varianzas no son homogéneas en todos los ámbitos estudiados, edad materna, trimestre de gestación, altitud a nivel del mar y departamentos indicando que las diferencias se deben a factores diferentes, considerándose adecuados (Figura 2).
Los niveles de hemoglobina y de la edad gestacional no siguieron una distribución normal.

La prevalencia de anemia en gestantes encontradas fue $28,0 \%$; su clasificación fue $25,1 \%$ para la anemia leve; $2,6 \%$ para la anemia moderada y $0,2 \%$ para la anemia grave. Con respecto a la edad, la prevalencia de anemia es menor en los mayores rangos de edad, aunque estas diferencias no fueron significativas. En los trimestres de gestación, la prevalencia de anemia es mayor conforme

Tabla 2. Prevalencia de anemia en gestantes atendidas en establecimientos del Ministerio de Salud del Perú, 2011

\begin{tabular}{|c|c|c|c|c|c|c|c|c|}
\hline \multirow{3}{*}{ Característica } & \multirow{3}{*}{ N. ${ }^{\circ}$} & \multirow{3}{*}{$\begin{array}{c}\text { Anemia } \\
\% \text { (IC95\%) }\end{array}$} & \multicolumn{6}{|c|}{ Anemia } \\
\hline & & & \multirow[b]{2}{*}{$\%$} & \multirow{2}{*}{$\begin{array}{l}\text { Leve } \\
\text { (IC95\%) }\end{array}$} & \multicolumn{2}{|c|}{ Moderada } & \multicolumn{2}{|r|}{ Grave } \\
\hline & & & & & $\%$ & (IC95\%) & $\%$ & (IC95\%) \\
\hline \multicolumn{9}{|l|}{ Edad (años) } \\
\hline $10-15$ & 6657 & $30,7 \quad(28,8-32.8)$ & 28,0 & $(26,0-30,0)$ & 2,5 & $(0,2-5,0)$ & 0,1 & $(0,0-2,6)$ \\
\hline $16-35$ & 253555 & $27,8 \quad(27,5-28,2)$ & 25,1 & $(24,8-25,4)$ & 2,5 & $(2,2-3,0)$ & 0,1 & $(0,0-0,6)$ \\
\hline $36-45$ & 27479 & $28,6 \quad(27,7-29,6)$ & 25,3 & $(24,3-26,3)$ & 3,0 & $(1,9-4,2)$ & 0,3 & $(0,0-1,5)$ \\
\hline \multicolumn{9}{|l|}{ Trimestre } \\
\hline Primer & 26123 & $15,6 \quad(14,5-16,7)$ & 14,1 & $(13,0-15,2)$ & 1,3 & $(0,2-2,6)$ & 0,1 & $(0,0-1,3)$ \\
\hline Segundo & 99737 & $26,2 \quad(25,7-26,8)$ & 23,7 & $(23,2-24,3)$ & 2,3 & $(1,7-3,0)$ & 0,1 & $(0,0-0,8)$ \\
\hline Tercer & 161831 & $31,0 \quad(30,7-31,5)$ & 27,8 & $(27,4-28,3)$ & 3,0 & $(2,5-3,5)$ & 0,2 & $(0,0-0,7)$ \\
\hline \multicolumn{9}{|l|}{ Altitud (m) } \\
\hline$<1000$ & 160224 & $20,3 \quad(19,9-20,8)$ & 19,4 & $(19,0-19,9)$ & 0,8 & $(0,4-1,3)$ & 0,05 & $(0,0-0,5)$ \\
\hline $1001-1500$ & 10741 & $23,3 \quad(21,7-25,0)$ & 21,8 & $(20,2-23,5)$ & 1,4 & $(0,0-3,3)$ & 0,09 & $(0,0-2,0)$ \\
\hline $1501-2500$ & 25876 & $18,5 \quad(17,4-19,6)$ & 17,5 & $(16,4-18,7)$ & 0,9 & $(0,0-2,1)$ & 0,0 & $(0,0-1,3)$ \\
\hline $2500-3500$ & 59932 & $38,4 \quad(37,8-39,1)$ & 33,1 & $(32,5-33,8)$ & 4,9 & $(4,2-5,8)$ & 0,3 & $(0,0-1,1)$ \\
\hline $3501-4000$ & 30918 & $57,0 \quad(56,4-57,8)$ & 46,9 & $(46,2-47,8)$ & 9,1 & $(8,1-10,2)$ & 0,9 & $(0,0-2,1)$ \\
\hline \multicolumn{9}{|l|}{ Departamentos } \\
\hline Amazonas & 4013 & $16,9 \quad(14,1-19,7)$ & 15,6 & $(12,8-18,5)$ & 1,1 & $(0,0-4,2)$ & 0,1 & $(0,0-3,2)$ \\
\hline Ancash & 14064 & $28,9 \quad(27,5-30,3)$ & 25,7 & $(24,3-27,2)$ & 3,0 & $(1,4-4,7)$ & 0,1 & $(0,0-1,8)$ \\
\hline Apurímac & 6445 & $40,8 \quad(38,9-42,7)$ & 30,8 & $(28,8-32,9)$ & 8,7 & $(6,4-11,0)$ & 1,1 & $(0,0-3,6)$ \\
\hline Arequipa & 13501 & $15,5 \quad(14,0-17,1)$ & 14,3 & $(12,8-16,0)$ & 1,0 & $(0,0-2,7)$ & 0,07 & $(0,0-1,8)$ \\
\hline Ayacucho & 12177 & $46,2 \quad(44,9-47,5)$ & 38,7 & $(37,4-40,2)$ & 6,9 & $(5,2-8,6)$ & 0,4 & $(0,0-2,2)$ \\
\hline Cajamarca & 15896 & $22,7 \quad(21,4-24,1)$ & 21,0 & $(19,7-22,5)$ & 1,5 & $(0,0-3,1)$ & 0,1 & $(0,0-1,7)$ \\
\hline Cusco & 19831 & $37,7 \quad(36,6-38,8)$ & 32,9 & $(31,8-34,1)$ & 4,5 & $(3,1-5,9)$ & 0,3 & $(0,0-1,7)$ \\
\hline Huancavelica & 6275 & $53,6 \quad(51,9-55,3)$ & 40,5 & $(38,7-42,5)$ & 11,7 & $(9,5-14,1)$ & 1,2 & $(0,0-3,7)$ \\
\hline Huánuco & 16384 & $30,8 \quad(29,5-32,1)$ & 27,4 & $(26,1-28,7)$ & 3,1 & $(1,7-4,7)$ & 0,2 & $(0,0-1,7)$ \\
\hline Ica & 7700 & $18,2(16,1-20,2)$ & 17,1 & $(15,1-19,2)$ & 0,9 & $(0,0-3,2)$ & 0,05 & $(0,0-2,3)$ \\
\hline Junín & 14419 & $34,4 \quad(33,1-35,7)$ & 29,8 & $(28,4-31,2)$ & 4,1 & $(2,6-5,8)$ & 0,4 & $(0,0-2,1)$ \\
\hline La Libertad & 15205 & $28,4 \quad(27,0-29,7)$ & 26,2 & $(24,9-27,6)$ & 2,0 & $(0,5-3,6)$ & 0,09 & $(0,0-1,7)$ \\
\hline Lambayeque & 8978 & $15,2 \quad(13,3-17,1)$ & 14,6 & $(12,8-16,6)$ & 0,4 & $(0,0-2,5)$ & 0,03 & $(0,0-2,1)$ \\
\hline Lima & 67168 & $22,9 \quad(22,3-23,6)$ & 21,8 & $(21,2-22,6)$ & 0,9 & $(0,2-1,7)$ & 0,04 & $(0,0-0,8)$ \\
\hline Loreto & 11565 & $18,8 \quad(17,2-20,5)$ & 18,0 & $(16,4-19,7)$ & 0,6 & $(0,0-2,5)$ & 0,1 & $(0,0-1,9)$ \\
\hline Madre de Dios & 2054 & $32,5 \quad(28,9-36,0)$ & 29,6 & $(26,0-33,2)$ & 2,4 & $(0,0-6,8)$ & 0,3 & $(0,0-4,7)$ \\
\hline Moquegua & 1030 & $23,6 \quad(18,3-28,9)$ & 21,4 & $(16,0-26,9)$ & 2,0 & $(0,0-8,1)$ & 0,1 & $(0,0-6,2)$ \\
\hline Pasco & 4483 & $39,2 \quad(36,9-41,5)$ & 34,3 & $(32,0-36,7)$ & 4,5 & $(1,7-7,4)$ & 0,3 & $(0,0-3,3)$ \\
\hline Piura & 11055 & $22,8 \quad(21,2-24,5)$ & 21,8 & $(20,2-23,5)$ & 0,9 & $(0,0-2,8)$ & 0,04 & $(0,0-1,9)$ \\
\hline Puno & 13250 & $51,0 \quad(49,8-52,2)$ & 45,0 & $(43,8-46,3)$ & 5,5 & $(3,9-7,2)$ & 0,4 & $(0,0-2,1)$ \\
\hline San Martín & 9475 & $22,4 \quad(20,6-24,2)$ & 20,8 & $(19,0-22,6)$ & 1,5 & $(0,0-3,5)$ & 0,07 & $(0,0-2,1)$ \\
\hline Tacna & 2385 & $21,6 \quad(18,0-25,1)$ & 19,6 & $(16,0-23,2)$ & 1,7 & $(0,0-5,7)$ & 0,2 & $(0,0-4,2)$ \\
\hline Tumbes & 2951 & $15,4 \quad(12,1-18,7)$ & 14,9 & $(11,6-18,3)$ & 0,4 & $(0,0-4,0)$ & 0,0 & $(0,0-1,0)$ \\
\hline Ucayali & 7387 & $18,2(16,1-20,3)$ & 17,2 & $(15,2-19,3)$ & 0,8 & $(0,0-3,1)$ & 0,07 & $(0,0-2,3)$ \\
\hline TOTAL & 287691 & $28,0 \quad(27,7-28,3)$ & 25,1 & $(24,9-25,5)$ & 2,6 & $(2,3-3,0)$ & 0,2 & $(0,0-3,7)$ \\
\hline
\end{tabular}


son mayores los trimestres; así mismo el incremento es mayor para la anemia leve que también muestra un comportamiento similar a la anemia en general, llegando al tercer trimestre en $27,8 \%$. Conforme a la altitud a nivel del mar, se pudo identificar que cuanto es mayor la altitud, es mayor también la prevalencia de anemia, siendo de $20,3 \%$ a los $1000 \mathrm{~m}$ de altitud y de $57,0 \%$ a más de $3500 \mathrm{~m}$ de altitud, este comportamiento tiene correspondencia con la anemia leve. Los departamentos de la sierra son los que tienen mayor prevalencia de anemia, tal es el caso de Huancavelica (53,6\%); Puno (51,0\%); Ayacucho (46,2\%), y Apurímac $(40,8 \%)$. Huancavelica es el departamento que tiene la prevalencia más alta tanto para la anemia en general, como también para la anemia leve $(40,5 \%)$ moderada $(11,7 \%)$ y grave $(1,2 \%)$ (Tabla 2$)$.

\section{DISCUSIÓN}

La prevalencia de anemia en gestantes atendidas en establecimientos del Ministerio de Salud del Perú fue de $28,0 \%$, valor muy similar a la cifra encontrada por ENDES $2011(27,8 \%)^{(7)}$ pero mayor a lo encontrado por Gonzales et al. $(18,1 \%)^{(8)}$. También se cuenta con otras prevalencias, que no son a nivel nacional, entre ellas las descritas en los hospitales de Pucallpa (70,3\%); Iquitos $(47,3 \%)$; La Oroya $(52,7 \%)$, o Huaraz $(7,1 \%)(15,16,17,22)$. Si hacemos una comparación con cifras de otros países como Argentina (2004) con $44 \%{ }^{(13)}$ y $30,5 \%$ (2007) $^{(6)}$; México, con informes que se mantienen en rangos entre 18,1 a $28,8 \%{ }^{(18,19)}$; Cuba, con cifras que oscilan entre 39,3 a $64,5 \%{ }^{(4,5,23)}$, o Chile donde oscila entre $10,9 \%$ a $13,4 \%{ }^{(3,20)}$, nos encontramos dentro de los rangos latinoamericanos. Sin embargo, las cifran en nuestro continente son menores a datos encontrados en países como India (87\%) o Bangladesh (74\%) ${ }^{(14)}$ los cuales presentan mayor prevalencia de anemia.

El presente estudio identificó una prevalencia de $25,1 \%$ de anemia leve; $2,6 \%$ de anemia moderada, y $0,2 \%$ de anemia grave, cifras más similares a la ENDES 2011, con $20,0 \%$ de anemia leve; $7,7 \%$ de anemia moderada, y $0,01 \%$ de anemia grave, que a las cifras encontradas por Gonzáles et al. ${ }^{8}$ ) con $16,6 \%$ de anemia leve; $1,4 \%$ de anemia moderada, y $0,1 \%$ de anemia grave. Se observa que la cifra en el presente estudio es mayor para la anemia leve frente a los otros estudios; menor a ENDES y mayor a la de Gonzáles en la anemia moderada y muy similar a los otros estudios en la anemia grave. Si bien los datos difieren en más de cinco puntos porcentuales, se puede considerar que los datos están dentro de los rangos esperados en este punto. Un estudio en México determinó anemia leve en $67,7 \%$; moderada en $27,9 \%$ y grave en $4,4 \%{ }^{(27)}$; mientras que en Cuba identificaron anemia leve en $75,8 \%$ y anemia grave en $2,2 \%{ }^{(4)}$, cifras superiores a las nuestras.

Las cifras halladas en este estudio solo pueden compararse con la Encuesta de Demografía y Salud (ENDES) y con el estudio de Gonzáles et al. (8) pues presentan datos nacionales; por las similitudes en el tamaño de la muestra y por la fuente de información, que en la encuesta del ENDES es poblacional, realizada a través de una muestra probabilística, de áreas, estratificada, bietápico e independiente en cada departamento (27) logrando una muestra para el año 2011 de 777 gestantes ${ }^{(26)}$ a nivel nacional. Mientras que en el estudio de Gonzáles et al. ${ }^{(8)}$ se tomaron 379816 registros de gestantes del Sistema Informático Perinatal de 43 centros asistenciales del Ministerio de Salud en un periodo de 10 años (2000 y 2010). En el presente estudio se ha utilizado un programa específico que toma toda la información de 33 direcciones de salud del Ministerio de Salud, que para el año 2011 fue de 318 436; de ellos, se tomaron 287691 (90,3\%), por lo que consideramos que el margen de error es menor y los datos son más consistentes. Nuestras diferencias también pueden deberse a que en el estudio de Gonzáles toma información de hospitales, mientras que nuestra información es en su mayoría de establecimientos de primer nivel $(98,2 \%)$ como puestos y centros de salud, teniendo un porcentaje menor de hospitales (Tabla 1).

Una limitante, por utilizar los niveles de hemoglobina para la determinación de la anemia por deficiencia de hierro, es que no es específica para esta causa, pero un estudio realizado en México (2009) determinó que el $94,2 \%$ de las anemias que se presentan en las gestantes son por deficiencias de hierro ${ }^{(25)}$, ello nos otorga un patrón de referencia aceptable, puesto que la anemia por deficiencia de hierro es la más prevalente.

En cuanto al promedio de hemoglobina en gestantes según el trimestre de gestación, se observó que la $\mathrm{Hb}$ es menor conforme son mayores los trimestres, pero solo en décimas de $\mathrm{g} / \mathrm{dL}$; datos diferentes a los de Escudero et al. (20) en los que los promedios disminuyeron en $1 \mathrm{~g} / \mathrm{dL}$ cada trimestre. Se sabe que durante la gestación los niveles de hemoglobina tienden a reducirse y esto es más marcado en los que tienen pocas reservas de hierro, puesto que la reducción de los niveles de hemoglobina es un indicador tardío de las reservas de hierro, y se sabe que durante la gestación no se puede cubrir las necesidades de hierro con solamente la dieta ${ }^{(6)}$. Consideramos que la reducción de la anemia en gestantes no fue muy marcada a pesar de recibir tratamiento, debido a que se encontraban con reservas bajas previas al inicio de la gestación, y por que el tiempo de ingesta que se ofrece a la gestante, es corto. 
Adicionalmente, los lugares con alta prevalencia de anemia son lugares donde existe alta deficiencia alimentaria ${ }^{(7)}$. De esta manera, los departamentos de la sierra son los que presentaron mayor prevalencia, siendo mayores en Huancavelica (53,6\%) y Puno (51,0\%). En Cusco encontramos una prevalencia de anemia en las gestantes de 37,7\%, mayor a lo encontrado por Gonzáles et al. ${ }^{(8)}$ que fue de 2,2\%; esto podría deberse a la diferencia de fuentes de información, y a su periodo de registro.

La Guía Nacional de Salud Sexual y Reproductiva ${ }^{(11)}$ indica que se debe administrar en forma profiláctica, a partir de las 16 semanas, dosis de $60 \mathrm{mg}$ de hierro elemental por día. Iniciar una vez confirmado el diagnóstico, con el doble de la dosis indicada para la administración profiláctica y reevaluar la hemoglobina en cuatro semanas; si se ha normalizado, continuar con esta dosis por tres meses y luego a dosis profiláctica hasta completar el puerperio. La OMS (12) refiere que cuando la prevalencia de anemia es mayor de $20 \%$ es de importancia moderada para la salud pública, y es grave cuando la prevalencia es mayor al $40 \%$. Actualmente, nuestro sistema de salud indica que a las mujeres en edad fértil y a las gestantes se les debe suplementar con sulfato ferroso, tarea más necesaria en la gestación puesto que los requerimientos extras no pueden ser obtenidos en la dieta; esta deficiencia se da en lugares donde los patrones culturales propios del Perú, que no tiene comparación en otro país, evitan el consumo adecuado de alimentos ricos en hierro, lo cual ocasiona prevalencias altas de anemia tanto en la mujer en edad fértil, como en la gestante. En los lugares donde la prevalencia es mayor al $30 \%$ debería haber suplementación con hierro, no solamente a las gestantes, sino a la población total, puesto que los patrones de anemia se inician desde antes del embarazo, ello reduciría las cifras de anemia, más aun si notamos las diferencias con el idioma, por lo que debería haber patrones de intervención diferenciados por zonas para las intervenciones en salud y nutrición.
Como se trata de un sistema de vigilancia pasiva, cuya cobertura equivale al $91,2 \%$ de los establecimientos de salud del Ministerio de Salud (28) y que representan $49,8 \%$ las gestantes peruanas en ese periodo, no es posible una plena extrapolación de los resultados, siendo muy aproximados por el tamaño de la población. A pesar que la recolección de la información es pasiva y regular por los establecimientos de salud del Ministerio y no fue realizado con fines de investigación, este sistema cuenta con procesos de control de calidad que disminuyen los sesgos propios de este tipo de sistema. Por ello, dado el tamaño de la población estudiada y el carácter de notificación obligatorio que tiene estos registros a nivel nacional, se espera que los resultados sean muy próximos a la realidad.

Como conclusión se puede indicar que la prevalencia de anemia en la gestante fue de $28,0 \%$, con una anemia leve de $25,1 \%$; moderada de $2,6 \%$, y grave de $0,2 \%$. Los niveles de hemoglobina aumentan conforme a la edad, y disminuyen conforme a la edad gestacional y altitud a nivel del mar. Así mismo, los departamentos de la sierra mantienen mayor prevalencia de anemia.

Contribuciones de autoría: OM participó en la concepción y diseño del artículo, análisis e interpretación de datos, y asesoría estadística. GG participó en la concepción y diseño del artículo, análisis e interpretación de datos, asesoría estadística y asesoría técnica o administrativa. JCB ha participado en el análisis e interpretación de datos, redacción del artículo y JS ha participado en obtención de financiamiento y asesoría técnica o administrativa. Todos los autores participaron en la revisión crítica del artículo y aprobación de su versión final.

Fuentes de financiamiento: Centro Nacional de Alimentación y Nutrición del Instituto Nacional de Salud. Lima, Perú.

Conflictos de interés: los autores declaran no tener conflictos de interés en la publicación de este artículo.

\section{REFERENCIAS BIBLIOGRÁFICAS}

1. Instituto Nacional de Estadística e Informática (INEI); Centro Nacional de Alimentación y Nutrición, Instituto Nacional de Salud (INS). Perfil nutricional y pobreza en Perú. Informe final 2009. Lima: INEI-INS; 2009.

2. Sánchez F, Trelles E, Terán R, Pedroso P. Nutrición, suplementación, anemia y embarazo. Rev Cubana Obstet Ginecol. 2001;27(2):141-5

3. Mardones F, Duran E, Villarroel L, Gattini D, Ahumada D, Oyarzún F, et al.
Anemia del embarazo en la provincia de Concepción, Chile: relación con el estado nutricional materno y el crecimiento fetal. Arch Latinoam Nutr. 2008;58(2):132-8

4. Sánchez F, Castañedo R, Trelles E, Pedroso P, Lugones M. Prevalencia de la anemia ferropénica en mujeres embarazadas. Rev Cubana Med Gen Integr. 2001;17(1):5-9

5. Villares I, Fernández J, Avilés M, Mediaceja O, Guerra T. Anemia y deficiencia de hierro en embarazadas de un área urbana del municipio Cien- fuegos. Rev Cubana Obstet Ginecol. 2006;32(1):1-8.

6. Casella A, Jelen A, Canalejo K, Aixalá M. Valores de referencia de la serie eritroide con tecnología del siglo XXI en embarazadas prevalencia de anemia. Acta Bioquim Clin Latinoam. 2007;41(1):47-50.

7. Instituto Nacional de Estadística e Informática (INEI). Perú: Encuesta Demográfica y de Salud Familiar 2011. Nacional y Departamental. Lima: INEI; 2012. 
8. Gonzales G, Tapia V, Gasco M, Carrillo C. Hemoglobina materna en el Perú: diferencias regionales y su asociación con resultados adversos perinatales. Rev Peru Med Exp Salud Publica. 2011;28(3):484-91.

9. Instituto Nacional de Estadística e Informática. Censos Nacionales 2007: XI de Población y VI de Vivienda. 2da Ed. Lima: INEI; 2008.

10. Parodi S. Evaluando los efectos del Seguro Integral de Salud (SIS) sobre la equidad en la salud materna en el contexto de barreras no económicas al acceso a los servicios. Lima: Grupo de Análisis para el Desarrollo; 2005.

11. Perú, Ministerio de Salud. Guías Nacionales de Atención Integral de la Salud Sexual y Reproductiva. Lima: Dirección General de Salud de las PersonasMINSA; 2004.

12. Organización Mundial de la Salud. Concentraciones de hemoglobina para diagnosticar la anemia y evaluar su gravedad. Ginebra: OMS; 2011.

13. Romero M, Cuenca E, López G, Rosales C. Anemia y control de peso en embarazadas. Rev Posgrado VIa Catedra Med. 2005;(143):6-8.

14. Gonzales GF, Steenland K, Tapia V. Maternal hemoglobin level and fetal outcome at low and high altitudes. Am J Physiol Regul Integr Comp Physiol. 2009;297()5:R1477-85.

15. Ramirez-Cardich M, Saito M, Gilman R, Escate L, Strouse J, Kabrhel $\mathrm{C}$, et al. Effect of maternal anemia at high altitude on infant hematocrit and oxygenation. Am J Trop Med Hyg. 2004;70(4):420-4.
16. Larocque R, Casapia M, Gotuzzo E, Gyorkos R. Relationship between intensity of soil-transmitted helminth infections and anemia during pregnancy. Am J Trop Med Hyg. 2005;73(4):783-9.

17. Gonzales G, Tapia V, Cerna J, Pajuelo A, Muñoz M, Carrillo C, et al. Características de la gestación, del parto y recién nacido en la ciudad de Huaraz, 2001-2005. Acta Med Per. 2006;23(3):137-43.

18. Casanueva E, De Regil L, Flores-Campuzano M. Anemia por deficiencia de hierro en mujeres mexicanas en edad reproductiva. Historia de un problema no resuelto. Salud Publica Mex. 2006;48(2):166-75.

19. Perichart O, Balas M, Schiffman E, Serrano M, Vadillo F. Impacto de la obesidad pregestacional en el estado nutricio de mujeres embarazadas de la Ciudad de México. Ginecol Obstet Mex. 2006;74(2):77-88.

20. Escudero L, Parra B, Restrepo S. Factores sociodemográficos y gestacionales asociados a la concentración de hemoglobina en embarazadas de la Red hospitalaria pública de Medellín. Rev Chil Nutr. 2011;38(4):429-37.

21. Kalaivani K. Prevalence \& consequences of anaemia in pregnancy. Indian J Med Res. 2009;130:627-33.

22. Becerra C, Gonzales G, Villena A, De la Cruz D, Florian A. Prevalencia de anemia en gestantes, Hospital Regional de Pucallpa, Perú. Rev Panam Salud Pública. 1998;3(5):285-92.

23. Prendes M, Baños A, Toledo O, Lescay O. Prevalencia de anemia en gestantes en un área de salud. Rev Cubana Med Gen Integr. 2000;16(1):25-30.
24. Mardones F, Rioseco A, Ocqueteau M, Urrutia M, Javet L, Rojas I, et al. Anemia en las embarazadas de la comuna de Puente Alto, Chile. Rev Méd Chile. 2003;131:520-5.

25. Iglesias-Benavides J, Tamez-Garza L, Reyes-Fernández I. Anemia y embarazo, su relación con complicaciones maternas y perinatales. Medicina Universitaria. 2009; 11(43):95-8.

26. Instituto Nacional de Estadística e Informática (INEI). Ficha técnica Encuesta Demográfica de Salud Familiar - ENDES 2010. Lima: INEI; 2010.

27. Olaiz G, Rivera J, Shamah T, Rojas R, Villalpando S, Hernández $M$, et al. Encuesta Nacional de Salud y Nutrición 2006. México: Instituto Nacional de Salud Pública; 2006.

28. Instituto Nacional de Salud (INS), Centro Nacional de Alimentación y Nutrición. Estado Nutricional en niños menores de 5 años y gestantes que acuden a los establecimientos de salud. Periodo Anual 2011. Lima: INS; 2011

Correspondencia: Oscar Fausto Munares García Dirección: Av. Tizón y Bueno 276. Lima 11, Perú.

Teléfono: 6176200 Anexo 6605/998719537 Correoelectrónico:omunares@ins.gob.pe
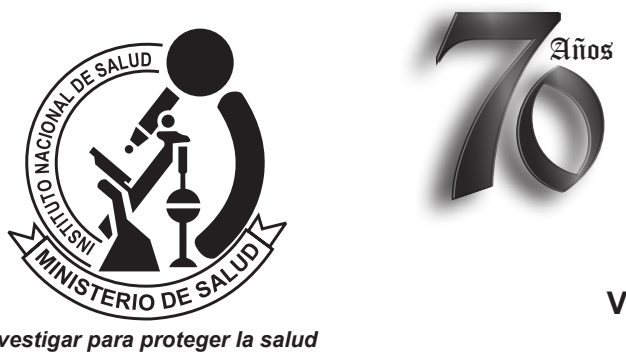

REVISTA PERUANA DE MEDICINA EXPERIMENTAL Y SALUD PÚBLICA CUMPLIENDO SUS METAS Y PROYECTÁNDOSE AL FUTURO

\section{Visite los contenidos de la revista en: www.ins.gob.pe/rpmesp}

